\title{
Confinement Analysis of Spherical Tokamak-Stellarator Hybrid Configurations with Simple Shaped Coils*)
}

\author{
Hidemasa OZEKI, Kozo YAMAZAKI, Hideki ARIMOTO, Tetsutarou OISHI, \\ Tatsuo SHOJI and Mikhail Ivanivich MIKHAILOV ${ }^{1)}$ \\ Nagoya University, Nagoya 464-8603, Japan \\ ${ }^{1)}$ Russian Research Centre “Kurchatov Institute”, Moscow, Russia
}

(Received 9 December 2011 / Accepted 27 June 2012)

\begin{abstract}
Six types of spherical tokamak-stellarator hybrid configurations with simple shaped coils "TOKASTAR", are proposed and analyzed in terms of magnetic flux surface, equilibrium and fast particle confinement. It was found that TOKASTAR can produce flux surfaces with low averaged aspect ratio. The averaged rotational transform are low but local value is relatively high, especially at the outboard side. In the equilibrium beta limit analysis it was clarified that TOKASTAR can achieve high equilibrium beta with relatively low plasma current. In the fast particle confinement analysis, it was found that the fraction of confined alpha particles in the reactor-sized TOKASTAR is $40 \sim 60 \%$.
\end{abstract}

(C) 2012 The Japan Society of Plasma Science and Nuclear Fusion Research

Keywords: tokamak, stellarator, TOKASTAR, magnetic flux surface, low aspect ratio, alpha particle confinement

DOI: $10.1585 /$ pfr.7.2403144

\section{Introduction}

Among magnetic confinement fusion systems tokamaks presently achieved higher total plasma confinement values than other systems. However, it is difficult to sustain steady tokamak operation because of disruption events and current-drive requirement. The effect of suppression of disruption due to superimposition of external helical field upon current-carrying plasma was confirmed experimentally [1]. Therefore it is important to study the effect of external helical field application to tokamak plasmas, especially in a compact low-aspect-ratio system with external outboard helical field because general stellarator devices have large aspect ratio.

Our research team proposed and has studied new magnetic configuration called "TOKASTAR" [2], which is spherical tokamak-stellarator hybrid configuration with simple shaped coils as seen in Fig. 1. In this figure $N$ is the toroidal mode number of helical coils. This configuration can produce low-aspect-ratio vacuum flux surface produced by currents of simple helical coils, so it is inexpensive to be built and suitable for doing basic fusion research relevant to spherical stellarator system and tokamak-stellarator hybrid system. Although the other compact hybrid systems have been proposed [3] so far, our TOKASTAR configuration is characterized by its simplicity and a small number of helical coils.

In the previous study of TOKASTAR only one typical coil shape shown in Fig. 1 is analyzed in detail [4]. To understand the plasma confinement properties

author'se-mail: yamazaki@ees.nagoya-u.ac.jp

*) This article is based on the presentation at the 21st International Toki Conference (ITC21).

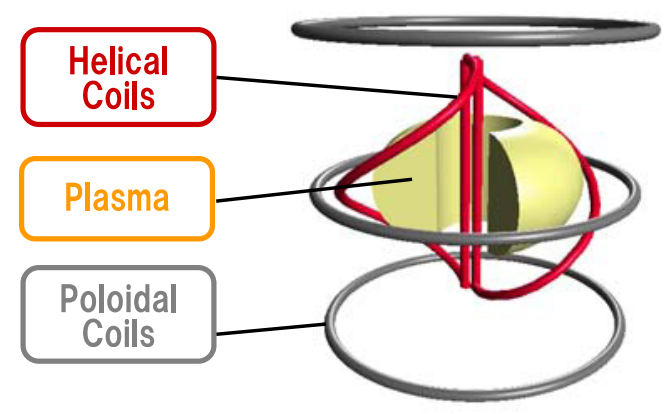

Fig. 1 Concept of previous $N=2$ TOKASTAR.

of TOKASTAR various types of helical field configuration should be studied and much more knowledge about TOKASTAR should be obtained. Therefore, in this paper new five TOKASTAR configurations are proposed, and a total of 6 types of TOKASTAR is analyzed in terms of fundamental magnetic properties and confinement capabilities, which include the shape of magnetic flux surface, rotational transform, equilibrium beta limit, and fast particle confinement. In addition to previous standard $N=2$ system, five new configurations are shown in Fig. 2: $N=1$ and $N=4$ with standard (straight) central filament, $N=$ 2 with central helix, $N=2$ with crescent cross-sectional configuration, and $N=2$ cylindrical coil system. Central helix configuration has helical central post. Crescent cross-sectional configuration has 3 inner poloidal coils at the central post to make the cross-sectional shape of the flux surfaces crescent. Cylindrical coil system has helical coils wound around cylinder, not on the sphere. 


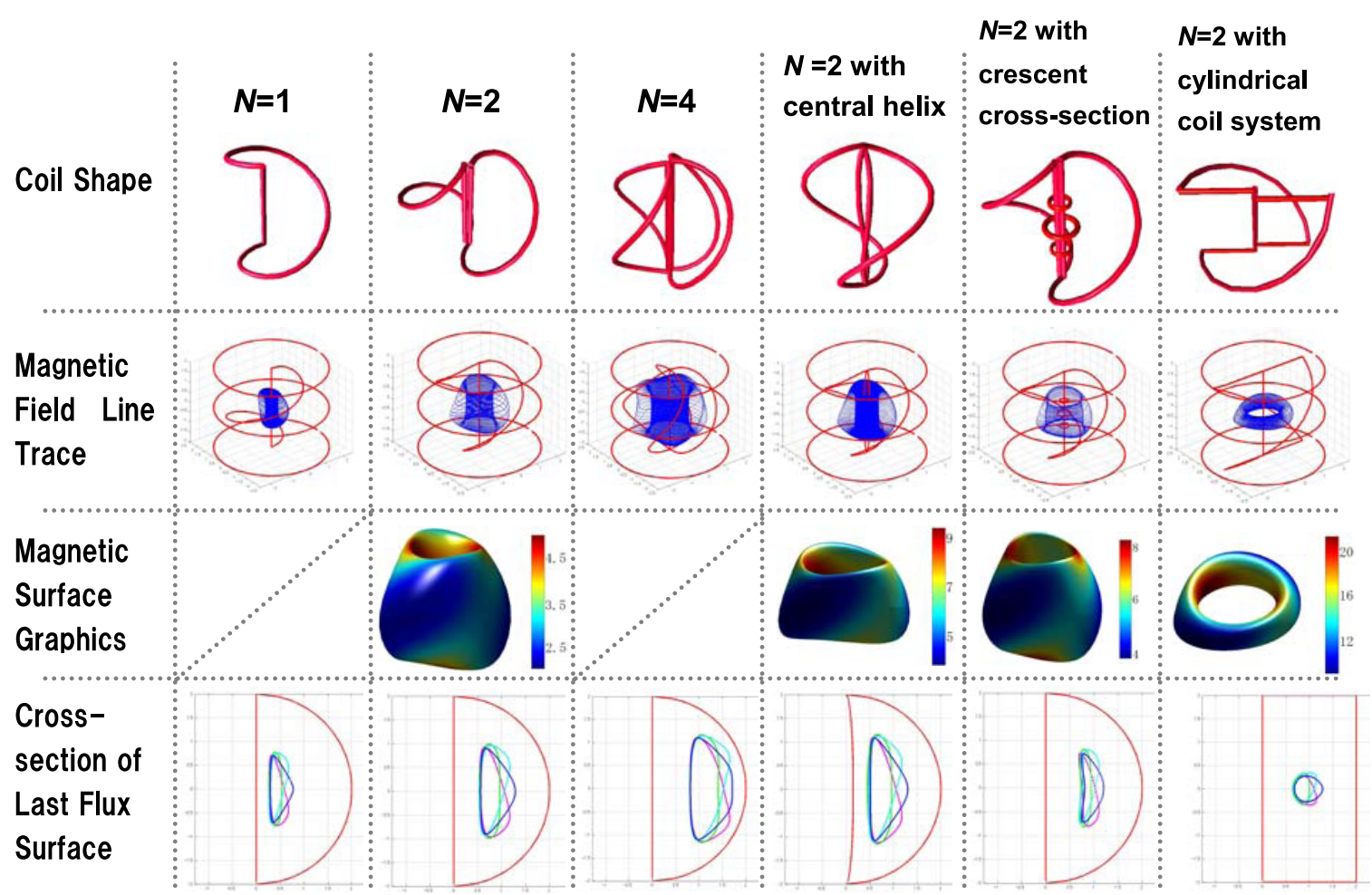

Fig. 2 Results of magnetic flux surface analysis of six TOKASTAR configurations.

\section{Method of Analysis}

TOKASTAR configurations were analyzed by computational simulation code. For magnetic flux surface analysis, we used the HSD code [2,5]. This code traces a magnetic field line produced by the coils and defines the vacuum magnetic flux surface. The guiding center orbit of single high-energy particle is also computed in this code. This code solves the particle drift equation

$$
\vec{v}=v_{/ /} \frac{\vec{B}}{B}+\frac{m}{q B^{3}}\left(\frac{1}{2} v_{\perp}^{2}+v_{/ /}^{2}\right) \vec{B} \times \nabla \vec{B} .
$$

In this computation, we used filament coil model. Plasma current and pressure were not considered when we used this code.

For equilibrium analysis, the VMEC code [6] was utilized. The finite-beta 3-dimenional equilibrium was solved with the results of flux surface analysis by this code. The computational procedure of VMEC is to minimize the total energy of plasma confined in a toroidal domain. The equilibrium states with finite-beta plasmas and the effects of plasma current in TOKASTAR were analyzed.

\section{Results of Vacuum Flux Surface Analysis}

\subsection{The shapes of last flux surfaces}

Vacuum magnetic flux surface analysis was carried out for 6 types of TOKASTAR with HSD code. The results of the last flux surface analysis for 6 types of TOKASTAR configurations are shown in Fig. 2, which shows the shapes of coils (first row from the top), the magnetic field lines on the last flux surfaces (second row), the 3D magnetic surface graphics (third row, colors show the relative magnetic field strength), and the cross-sections of last flux surface per $1 / 4$ toroidal period (fourth row). It should be noted that these flux surfaces are produced by only helical coil currents and poloidal coil currents. The shape parameters of these last flux surfaces are shown in Table 1. As you can see in this table, TOKASTAR can produce vacuum flux surfaces with low $\left\langle A_{\mathrm{p}}\right\rangle$ and high elongation, where $\left\langle A_{\mathrm{p}}\right\rangle=$ $R_{0} /\langle\sqrt{a \cdot b}\rangle, R$ : major radius, $a$ : horizontal minor radius $b$ : vertical minor radius, $\kappa=b / a$. Magnetic axis radius normalized by coil radius $R_{\mathrm{ax}} / R_{\mathrm{c}}$ is also shown. Here, the elongation was roughly estimated from the averaged ratio of vertical width and horizontal width of four magnetic surfaces within one pitch interval.

\subsection{Rotational transform at the last flux sur- faces}

Averaged rotational transform on the whole last flux surface $\langle t\rangle$ and maximum local rotational transform values at the last flux surfaces $t_{\max }$ are checked [2,4] for TOKASTAR. The results are shown in Table 1. The $\langle t\rangle$ value is quite low and is order of $10^{-2}$. However the $t_{\max }$ values are very high in comparison with the averaged values and are approximately $1 \sim 0.1$. These locally strong rotational transform were obtained at the outboard side of torus, where the helical coils are close to the last flux surface. This tendency obtained in all of TOKASTAR. Because the curvature at the outboard side of torus is generally bad and plasma tends to be unstable here, these $t$ pro- 
Table 1 Shape parameters of flux surface and rotational transform for each TOKASTAR.

\begin{tabular}{|l|c|c|c|c||c|c|}
\hline & $\begin{array}{c}A_{\mathrm{p}} \\
\left(=R_{0} / a\right)\end{array}$ & $\begin{array}{c}\kappa \\
(=b / a)\end{array}$ & $\begin{array}{c}\left\langle A_{\mathrm{p}}\right\rangle \\
\left(=R_{0} / \sqrt{a b}\right)\end{array}$ & $R_{\mathrm{ax}} / R_{\mathrm{c}}$ & $(\boldsymbol{t})$ & $\boldsymbol{t}_{\max }$ \\
\hline$N=1$ & 2.3 & 3.6 & 1.2 & 0.6 & 0.015 & 0.37 \\
\hline$N=2$ ( previous $)$ & 2.7 & 3.0 & 1.6 & 1.1 & 0.023 & 0.67 \\
\hline$N=4$ & 3.0 & 2.8 & 1.8 & 1.5 & 0.029 & 1.00 \\
\hline$N=2$ central helix & 2.5 & 3.3 & 1.4 & 1.1 & 0.017 & 0.66 \\
\hline$N=2$ cylindrical & 3.3 & 1.2 & 3.1 & 1.1 & 0.025 & 0.49 \\
\hline$N=2$ crescent & 5.3 & 4.1 & 2.6 & 1.1 & 0.015 & 0.45 \\
\hline
\end{tabular}

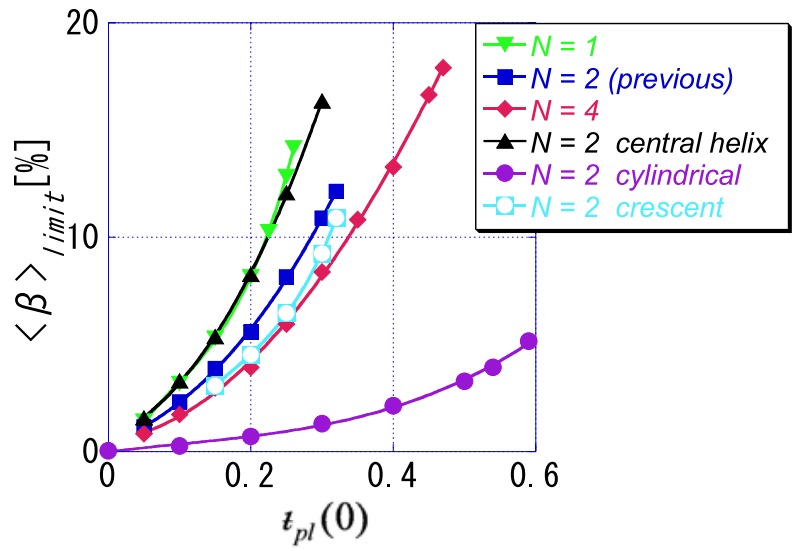

Fig. 3 Result of equilibrium beta limit of TOKASTAR.

file tendencies may be helpful to achieve high beta plasma by the short line-tying stabilization in the TOKASTAR.

\section{Results of Equilibrium Beta Limit Analysis}

In the equilibrium analysis with VMEC code, we studied equilibrium beta limit of TOKASTAR configurations. This beta limit is determined by the convergence condition of VMEC code and the critical Shafranov shift larger than half plasma radius. The rotational transform of current carrying Tokastar is defined by the summation $\mathrm{f} \mathrm{ex-}$ ternal vacuum transform and the plasma current transform $t_{\mathrm{pl}}$. The result of equilibrium beta limit analysis is shown in Fig. 3. The vertical axis indicates the volume-averaged equilibrium beta limit $\langle\beta\rangle_{\text {limit }}$. The horizontal axis indicates the value of rotational transform at the plasma center $t_{\mathrm{pl}}(0)$ produced by plasma current. The radial dependence of the plasma current was assumed

$$
t_{\mathrm{pl}}(S)=t_{\mathrm{pl}}(0)\left(1-S+1 / 3 S^{2}\right),
$$

where $s$ is normalized toroidal flux and $s \sim \rho^{2}$. The pressure profile was assumed as parabolic one like $1-s$.

As seen in Fig. 3, $N=1$ and $N=2$ with central helix configuration achieved higher beta with low plasma current than the other TOKASTAR. As Table 1 and Fig. 3 are compared, we found that the configurations with lower $\left\langle A_{\mathrm{p}}\right\rangle$ can achieve higher beta limit.

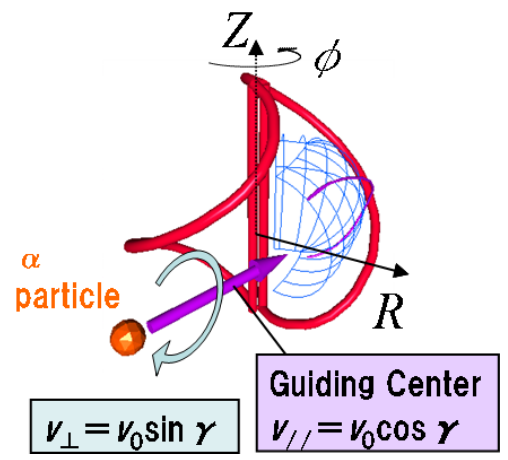

Fig. 4 Schematic view of single fast particle confinement analysis.

\section{Results of Fast Particle Confine- ment Analysis}

\subsection{Condition of calculation and input pa- rameters}

In this chapter the confinement capability of single fast particle of TOKASTAR without plasma current is analyzed with the same value of normalized Larmor radius of alpha particle $\rho_{\alpha} / R_{0}$ in the SlimCS tokamak [7]. According to the design parameters of SlimCS, its major radius $R_{0}$ is $5.5 \mathrm{~m}$ and its toroidal magnetic field at the axis is $6 \mathrm{~T}$. As an alpha particle with the mass of $6.664 \times 10^{-27} \mathrm{~kg}$, the charge of $3.204 \times 10^{-19} \mathrm{C}$ and the energy of $3.52 \mathrm{MeV}$ moves in the magnetic field of $6 \mathrm{~T}$, the Larmor radius $\rho_{\alpha}$ $(=m v / q B)$ is approximately $4.5 \times 10^{-2} \mathrm{~m}$ and then $\rho_{\alpha} / R_{0}$ is $8.2 \times 10^{-3}$.

In the case of TOKASTAR configurations calculated here, the input parameters were set to the following values. The mass and the charge of alpha particle is the same mentioned above. The radius of spherical helical coils of TOKASTAR is $2 \mathrm{~m}$ and the magnetic field strength at $R=$ $1 \mathrm{~m}$ is $0.4 \mathrm{~T}$. The initial velocity of the particle $v_{0}$ is set to $1.58 \times 10^{5} \mathrm{~ms}^{-1}$ so that $\rho_{\alpha} / R_{0}$ becomes $8.2 \times 10^{-3}$. This is the same value as that of SlimCS.

The schematic model of the particle orbit analysis is shown in Fig. 4. The guiding center orbit of alpha particle was calculated changing its initial position $R$ and pitch angle $\gamma$. The particles were started with $Z=0$ and $\varphi=0$ (helical coil position on the equatorial plane). The calcula- 


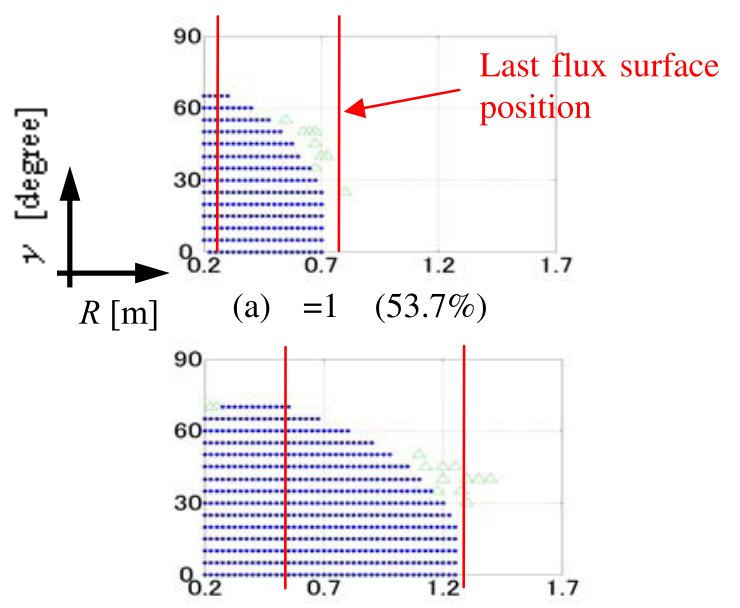

(b) $=2 \quad(57.4 \%)$

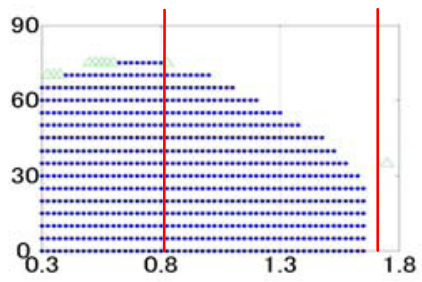

(c) $=4 \quad(59.1 \%)$

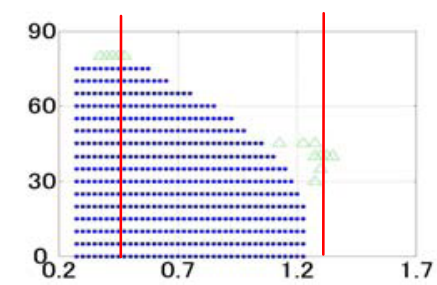

(d) $=2$ central helix $(60 \%)$

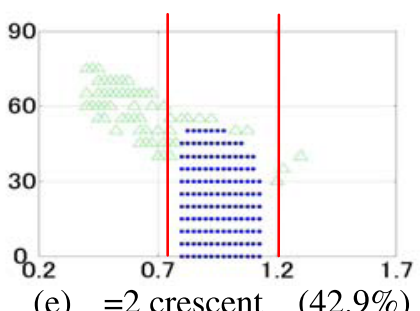

(e) $=2$ crescent $(42.9 \%)$

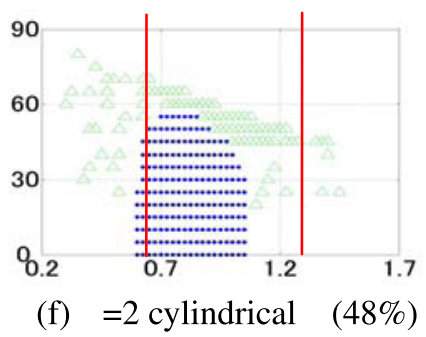

Fig. 5 Computational results of single fast particle confinement for each TOKASTAR. The blue dots in the figures indicate the confined passing particles and the green triangles indicate the confined trapped particles. The numbers in the parentheses indicates the rate of confined particles among those which started to move inside the last flux surface (area ratio of blue and green dots to the rectangular area between red lines). tion of the particle orbit was continued unless the particle moved $1 \mathrm{~km}$ (correspond to about 160 turns to the toroidal direction at $R=1 \mathrm{~m}$ ) or lost from each TOKASTAR coil boundary system, which means the limiter shape is sphere or cylinder depending on the coil system.

\subsection{Results of fast particle confinement cal- culation}

The results of fast particle confinement calculation are shown in Fig. 5. In these figures, horizontal axis $R$ indicates the initial positions where the particles started to move. The vertical axis $\gamma$ indicates the initial pitch angle for the particle initial velocity. The blue dots indicate the passing particles and the green triangles indicates the trapped particles, which means these blue and green points indicate particle confined in the coil systems. The numbers in the parentheses indicates the fraction of confined particles among those which started to move inside the last flux surface. In other words, this is the ratio of blue and green dot area to the rectangular area defined by red lines. This fraction of confined particles of TOKASTAR are about $40 \sim 60 \%$. The particles with large vertical velocity component tend not to be confined in TOKASTAR coil systems. It can be considered that this is caused by the weak averaged rotational transform of TOKASTAR. To improve that large plasma current is needed, or some optimizing methods of configurations should be explored.

\section{Summary}

In this study six types of TOKASTAR are proposed and evaluated by using HSD code and VMEC code in terms of magnetic flux surface, rotational transform, equilibrium beta, and fast particle confinement.

We found that TOKASTAR configurations can produce flux surfaces with low $\left\langle A_{\mathrm{p}}\right\rangle$. As concerns rotational transform, averaged value is very low but local value is very high, especially at the outboard side of torus. In the equilibrium beta limit analysis it was clarified that TOKASTAR can achieve high equilibrium beta with low plasma current. From the view-point of fast particle confinement, we found that the fraction of confined particles of TOKASTAR is about $40 \sim 60 \%$ when they start to move inside the closed flux surfaces. The particles with large perpendicular velocity tend not to be confined.

[1] WVII-A TEAM, Nucl. Fusion 20, 1093 (1980).

[2] K. Yamazaki and Y. Abe, Nagoya Research Report IPPJ718 (1985).

[3] P.E. Moroz, Nucl. Fusion 37, 1045 (1997).

[4] Y. Taira and K. Yamazaki et al., Plasma Fusion Res. 5, S1025 (2010).

[5] K. Yamazaki et al., Fusion Technol. 21, 147 (1992).

[6] S.P. Hirshman and W.I. van Rij, Comp. Phys. Comm. 43, 143 (1986).

[7] K. Tobita et al., Nucl. Fusion 49, 075029 (2009). 